\title{
Morphology Control by the Centrifugation Method for Bulk Heterojunction Solar Cells
}

\author{
T. Kato, N. Hagiwara, E. Suzuki, Y. Nasu, S. Izawa, and K. Tanaka
}

\begin{abstract}
A number of studies have been reported thin-film bulk heterojunction solar cells using p-type semiconducting materials as electron donors and n-type semiconducting materials as electron acceptors. Moreover, some studies have reported that controlling the morphology of the photoactive layer is important. In this study, we report a novel method for film formation utilizing centrifugation to control the morphology of the photoactive layer in thin-film solar cells. By employing this method, we observed high performance. Furthermore, the photoactive layer formed by the centrifugation method resulted in suitable phase-separated structures for charge separation and charge transfer, which were similar to a mutual penetration structure. We also fabricated thin-film bulk heterojunction solar cells using this method and obtained a performance that was 2.2 times greater than that obtained by the conventional spin-coating method using a p-type semiconducting polymer and Ti(IV) isopropoxide as the electron donor and electron acceptor, respectively.
\end{abstract}

Index Terms-Morphology control, thin film solar cells, bulk heterojunction solar cells, phase separation, photoactive layer, photovoltaic cells.

\section{INTRODUCTION}

Organic photovoltaic devices constitute a rapidly emerging technological area because of their promising properties, including flexibility, light weight, transparency, low cost, and large-area manufacturing compatibility [1]-[6]. In recent years, dye-sensitized solar cells and organic thin-film solar cells have been reported as next-generation developments in this field. Furthermore, the performance of perovskite-sensitized solar cells has rapidly increased. As organic thin-film solar cells, bulk heterojunction organic thin-film solar cells using organic semiconducting polymers have been one of the most extensively studied research topics. Typically, in these devices, the photoactive layers are composed of p-type semiconducting polymers and n-type fullerene derivatives such as [6,6]-phenyl-C61 or C71-butyric acid methyl ester ([60]PCBM or [70]PCBM) [7]-[10].

However, as fullerene and its derivatives are sensitive to air,

Manuscript received January 10, 2016; revised February 28, 2016. This work was supported, in part, by a Grant-in-Aid for Young Scientists (B) (25871029) from the Japanese Society for the Promotion of Science (JSPS), the Nippon Sheet Glass Foundation for Materials Science and Engineering, and the Tochigi Industrial Promotion Center. The National Institute of Technology, Oyama College, also assisted in meeting the publication costs of this article.

T. Kato, E. Suzuki, Y. Nasu, S. Izawa, and K. Tanaka are with Department of Mechanical Engineering, National Institute of Technology, Oyama College, 771 Nakakuki, Oyama, Tochigi 323-0806, Japan (e-mail: Kato_t@oyama-ct.ac.jp).

N. Hagiwara is with Ashizawa Finetech Ltd., 2-1-4 Hitotonoya, Oyama, Tochigi 323-0034, Japan. stable, alternative electron acceptors are needed. In this regard, several studies have described new types of bulk heterojunction thin-film solar cells utilizing conjugated polymers and n-type inorganic materials as n-type semiconductors [11]-[13]. These studies have inspired the development of fullerene-free, organic thin-film solar cells. A previous study reported the formation of fullerene-free organic-inorganic thin-film solar cells utilizing Ti(IV) isopropoxide as the electron acceptor [14], and our group demonstrated the importance of controlling the phase-separated structure in the photoactive layer with the aim of obtaining a high energy conversion efficiency [15]. We have demonstrated that the molecular structure and size of $\mathrm{Ti}$ alkoxide are effective for controlling the morphology of the photoactive layer. To control the morphology using the molecular structure, this method (this method means utilizing molecular structure) effectively restricted the formation of the photoactive layer [15]. However, if the molecular structure is used to control the morphology, all parts of the photoactive layer will change uniformly. Hence, this method cannot control the morphology in the direction of both electrodes. In other words, this method cannot form a $\mathrm{p}-\mathrm{n}$ gradient structure into the photoactive layer. Moreover, some studies have revealed that the use of thermal annealing and some additives as well as several solvents are essential for the formation of a suitable photoactive layer [16], [17]. The use of either of these methods will result in a uniform change in the morphology in all parts of the photoactive layer. However, these methods cannot control the morphology in the direction of both electrodes for the efficient transfer of free carriers. On the other hand, some studies have demonstrated that centrifugal force is effective for changing the morphology of a polymer solution or the solution containing titanium compounds. In this study, we use the centrifugation method and demonstrate morphology control in the direction of both electrodes in the formation of a $p-n$ gradient structure [18], [19].

\section{EXPERIMENTAL}

Poly(3-hexylthiophene-2,5-diyl) (P3HT, electron donor) and $\mathrm{Ti}(\mathrm{IV})$ isopropoxide as the p-type and n-type semiconducting polymers, respectively, were purchased from Sigma-Aldrich. Fig. 1 shows the molecular structures of these materials and their energy diagram. Indium-tin oxide (ITO)-coated glass substrates (15 $\Omega$ per square) were patterned for the fabrication of the solar cells. First, these substrates were successively washed by ultrasonication in water, acetone, and isopropyl alcohol for $15 \mathrm{~min}$ each and then dried under a stream of dry air. 


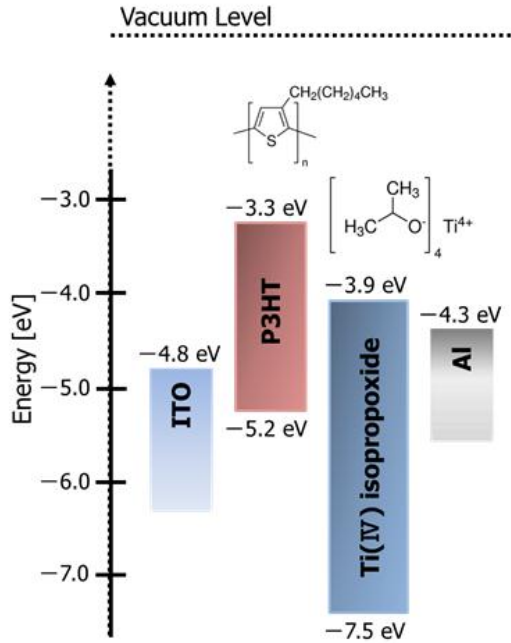

Fig. 1. Chemical structures of P3HT and Ti(IV)isopropoxide and energy diagram.

Second, the washed substrates were further treated with a UV-O 3 cleaner (Filgen, Model UV253E) for 20 min. Third, a photoactive layer of P3HT- Ti(IV) isopropoxide (1:2 wt \%) was centrifugation-coated on the ITO substrates at 2000 to $6000 \mathrm{rpm}$ for $20 \mathrm{~s}$ from a chlorobenzene solution; for comparison, the same photoactive layer was also coated by the conventional spin-coating method. Fig. 2 shows the centrifugation film-coating system fabricated in this study.

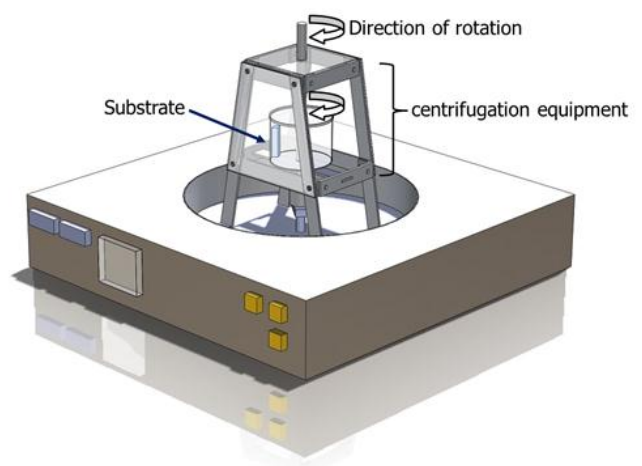

Fig. 2. Schematic diagram of the centrifugation film-coating system

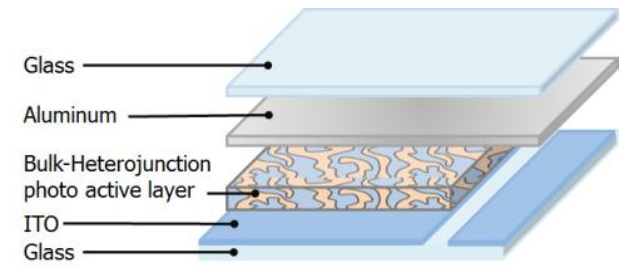

Fig. 3. Structure of thin-film bulk heterojunction solar cells.

The manufactured centrifugation equipment was attached to the rotating part of a spin-coater (KYOWA RIKEN Ltd; K359S-1). The radius of gyration from the center to the substrate was adjusted to $10 \mathrm{~cm}$. The spin-coating method was used as commonly available reference method that could be conducted under the same conditions as centrifugation coating. Moreover, to promote the hydrolysis of Ti(IV) isopropoxide, the substrate with the photoactive layer was placed overnight in a humidistat bath maintained at $80 \%$ humidity. Moreover, all photoactive layers were dried under dark conditions. The counter electrodes, aluminum $(200 \mathrm{~nm})$ was deposited by vacuum evaporation. The photoactive layer and counter electrodes were laminated using an epoxy resin.
Fig. 3 shows the device structure.

The current density-voltage $(\mathrm{J}-\mathrm{V})$ characteristics were measured using a direct-current voltage and a current source-monitor under illumination with AM1.5G simulated solar light (San-Ei Electric, XES-40S1) at $100 \mathrm{~mW} / \mathrm{cm}^{2}$. The light intensity was corrected against a calibrated silicon photodiode reference cell (Bunko-Keiki, BS-520). On the other hand, to measure the transmittance and surface roughness of the photoactive layers, a substrate with a photoactive layer was placed in a humidistat bath maintained at $80 \%$ humidity for 3 days in order to rapidly advance the hydrolysis for the crystallization of $\mathrm{Ti}(\mathrm{IV})$ isopropoxide. Moreover, we also prepared a substrate with a photoactive layer, which was not hydrolyzed, for comparison. After coating each photoactive layer on a glass substrate by each coating method, UV-Vis-near-IR spectra (light transmittance) were recorded on a calibrated UV spectrophotometer (Shimadzu, UV-1800). The surface state and roughness of the photoactive layer were measured using a stylus profiler (Bruker, DEKTAK XT-E).

\section{RESULTS AND DISCUSSION}

We fabricated bulk heterojunction solar cells and investigated their photovoltaic performance [20]-[23]. Figure 4 shows the photocurrent density of the bulk heterojunction solar cells with photoactive layers of different thicknesses, fabricated by different film formation methods.

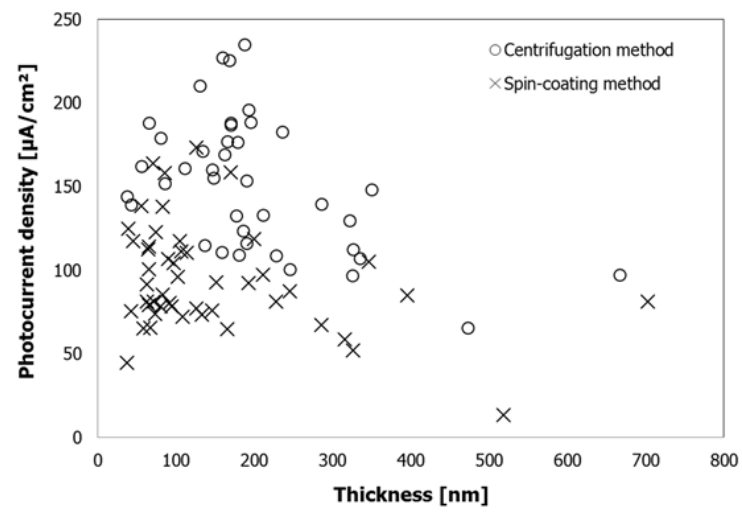

Fig. 4. Relationship between the current density and thickness of photoactive layers.

TABLE I: PERFORMANCE PARAMETERS OF DEVICES FORMED USING DIFFERENT FILM FORMATION METHODS

\begin{tabular}{lcc}
\hline \hline & $\begin{array}{l}\text { Centrifugation } \\
\text { method }\end{array}$ & $\begin{array}{l}\text { Spin-coating } \\
\text { method }\end{array}$ \\
\hline $\begin{array}{l}\text { Photocurrent density } \\
{\left[\mu \mathrm{A} / \mathrm{cm}^{2}\right]}\end{array}$ & 235 & 158 \\
Open circuit Voltage [V] & 0.45 & 0.44 \\
Fill factor & 0.45 & 0.31 \\
Power conversion efficiency [\%] & 0.047 & 0.021 \\
\hline \hline
\end{tabular}

The photocurrent density values of the photoactive layers with thicknesses of around 100-300 nm formed by the centrifugation method were greater than those obtained by the conventional spin-coating method. In particular, a $200 \mathrm{~nm}$ thick photoactive layer exhibited a current density of $235 \mu$ $\mathrm{A} / \mathrm{cm}^{2}$ and fill factor of 0.45 , which was approximately 1.5 
times that obtained using the spin-coating method $(158 \mu$ $\left.\mathrm{A} / \mathrm{cm}^{2}, 0.31\right)$. The corresponding performance parameters are listed in Table I.

The specific gravities of $\mathrm{P} 3 \mathrm{HT}$ and $\mathrm{Ti}(\mathrm{IV})$ isopropoxide are $1.1 \mathrm{~g} / \mathrm{cm}^{3}$ and $0.96 \mathrm{~g} / \mathrm{cm}^{3}$, respectively [24]. As a result, P3HT preferentially undergoes phase formation, and initially, the ITO side, which was subjected to centrifugal force, and the photoactive layer form a structure similar to that of a mutual penetration structure. A phase-separated structure in the photoactive layer of a bulk heterojunction solar cell is an important factor for the overall photovoltaic performance [25]-[27]. That is, we consider that by using the centrifugation method, the resulting morphologies exhibited adequate continuity in the direction of both electrodes for the charge transfer of free carriers. Table II lists the relationship between the phase-separated models and the photocurrent density values of the devices.

TABLE II: PHASE-SEPARATION MOdELS OF THE PHOTOACTIVE LAYERS IN

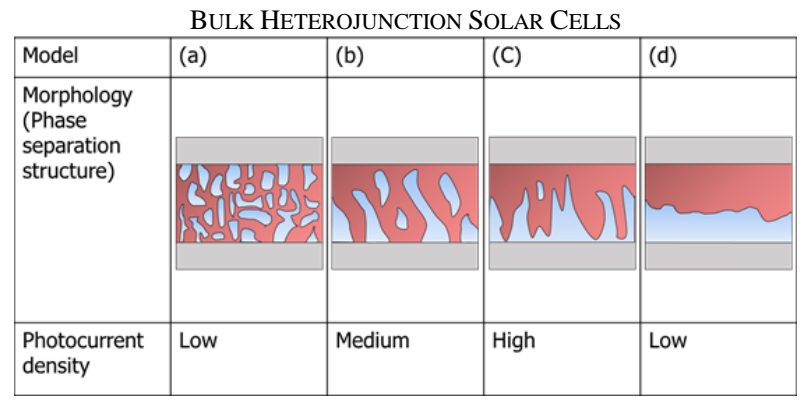

In model (a), isolated respective phases were formed, which would inhibit the charge transfer of free carriers to both electrodes. Ideally, a continuous phase structure is required in the direction of both electrodes, as indicated by models (b) and (c) in Table II. In consideration of this analysis, the photoactive layers, which rapidly advanced hydrolysis for the crystallization of $\mathrm{Ti}(\mathrm{IV})$ isopropoxide, were subjected to transmittance and surface roughness measurements. If a lot of Ti exists at the surface, the transmittance will decrease owing to reflection of the crystallized $\mathrm{Ti}$, and the surface roughness will also increase owing to the crystallized Ti. Fig. 5 shows the transmittance spectra of the photoactive layers.
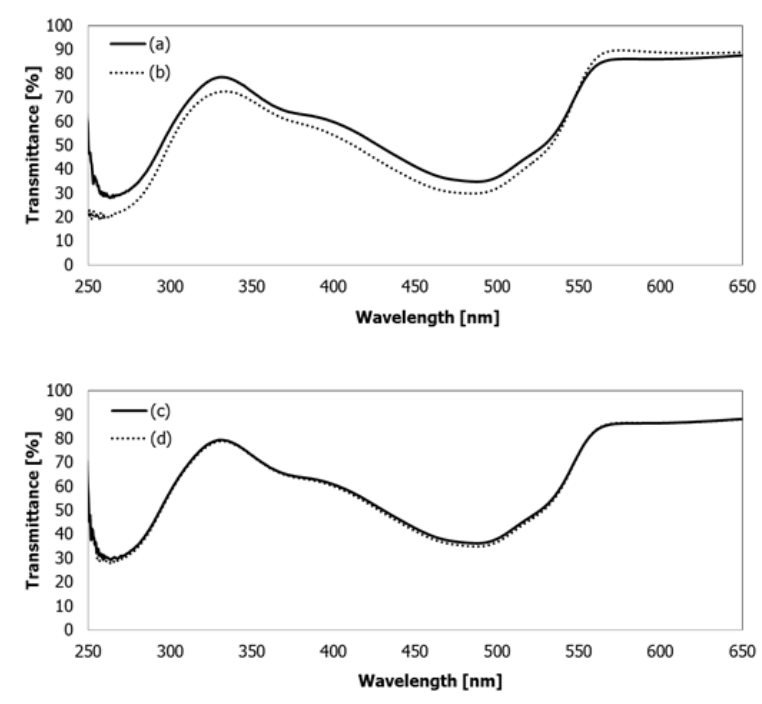

Fig. 5. Transmittance spectra of photoactive layers.

The light transmittance of the photoactive layer decreased, which can be observed by the change in the spectrum (b) to the spectrum (a) in Fig. 5, when using the centrifugation method. In contrast, when the conventional spin-coating method was used, the transmittance value did not change, as indicated by the change in spectrum (d) from the spectrum (c) in Fig. 5. That is, this phenomenon is indicative of the deposition of $\mathrm{Ti}$ isopropoxide, which has a lower specific gravity, to the upper layer of the photoactive layer. Titanium oxide was formed by hydrolysis with moisture at the surface of the photoactive layer, and then the titanium oxide layer reflected the incident light; hence, the transmittance of the thin film decreased. Moreover, the surface state of the photoactive layer before and after the crystallization of Ti(IV) isopropoxide, which was fabricated by each film formation method, was investigated. The average surface roughness of the photoactive layer formed by the centrifugation method was $3.22 \mathrm{~nm}$ and $59.89 \mathrm{~nm}$ before and after crystallization, respectively, as shown in Fig. 6 and Table III.

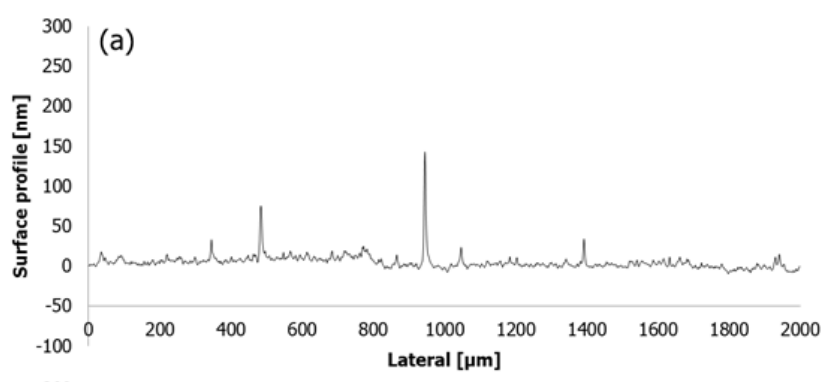

(b)

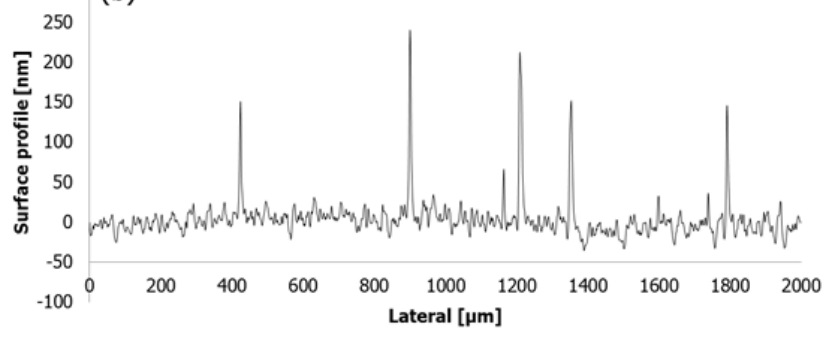

Fig. 6. Surface states of photoactive layers fabricated by the centrifugation method before and after crystallization of Ti(IV)isopropoxide. (a) Before crystallization, (b) after crystallization.Magnetization as a function of applied field.

TABLE Ш: SURFACE ROUGHNESS BEFORE AND AFTER CRYSTALLIZATION OF TI(IV) ISOPROPOXIDE

\begin{tabular}{lll}
\hline \hline Method & $\begin{array}{l}\text { Centrifugation } \\
\text { method }\end{array}$ & $\begin{array}{l}\text { Spin-coating } \\
\text { method }\end{array}$ \\
\hline Before crystallization & $3.22 \mathrm{~nm}$ & $2.40 \mathrm{~nm}$ \\
After crystallization & $59.89 \mathrm{~nm}$ & $4.36 \mathrm{~nm}$ \\
\hline \hline
\end{tabular}

The average surface roughness values of the photoactive layer formed by the conventional spin-coating method were $2.40 \mathrm{~nm}$ and $4.36 \mathrm{~nm}$ before and after crystallization, respectively, as shown in Fig. 7 and Table III.

In other words, the increase in surface roughness indicated an increase in the amount of the crystallized titanium oxide. Next, we investigated the relationship between centrifugation force and power generation. Figure 8 shows the relationship between the centrifugation force and photocurrent density of the solar cells.

The spin speed was fixed at $2000 \mathrm{rpm}$ to $6000 \mathrm{rpm}$ with increments of $500 \mathrm{rpm}$, and the experiment was performed at a radius of gyration of $10 \mathrm{~cm}$. Upon applying a centrifugal force of approximately $1000 \mathrm{G}$, the highest photocurrent 
density was observed. When a centrifugal force of less than approximately $1000 \mathrm{G}$ was applied, a phase-separated structure was formed in isolated domains, similar to that observed when the spin-coating method was utilized in model (a) and Table II. In this case, the respective phases were isolated, which would inhibit the charge transfer of free carriers to both electrodes. In contrast, when a centrifugal force of approximately greater than 1000G was applied, a phase-separated structure was formed, similar to the formation of a bilayer structure in model (d) in Table II. That is, in this case, the production of a sufficient number of $p-n$ junction surfaces for charge separation would be inhibited in the photoactive layer. On the other hand, when a centrifugal force of approximately $1000 \mathrm{G}$ was applied to the photoactive layer, a continuous phase structure in the direction of both electrodes was observed for the efficient charge transfer of free carriers.

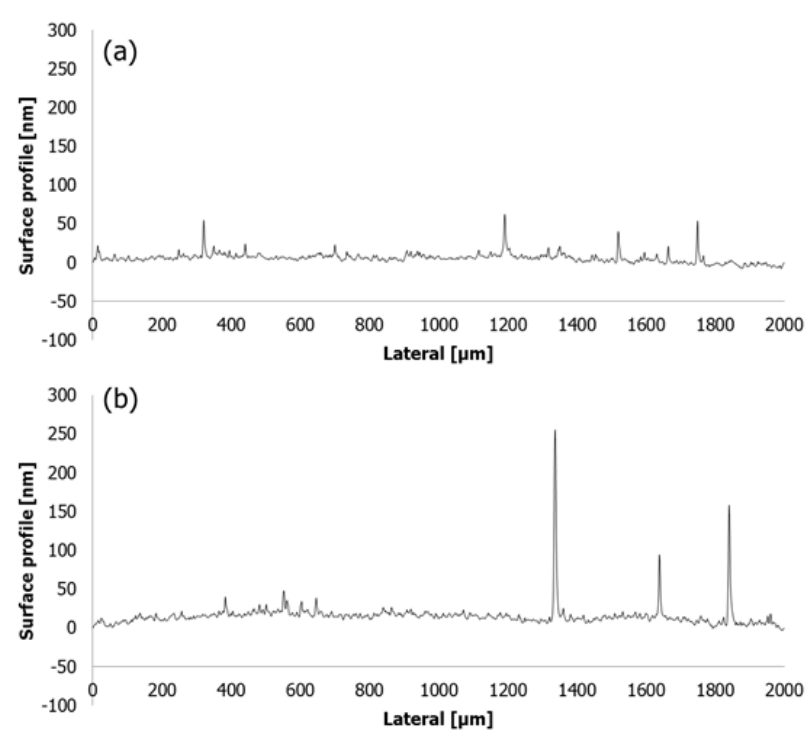

Fig. 7. Surface states of photoactive layers fabricated by the spin-coating method before and after crystallization of Ti(IV) isopropoxide. (a) Before crystallization, (b) after crystallization.

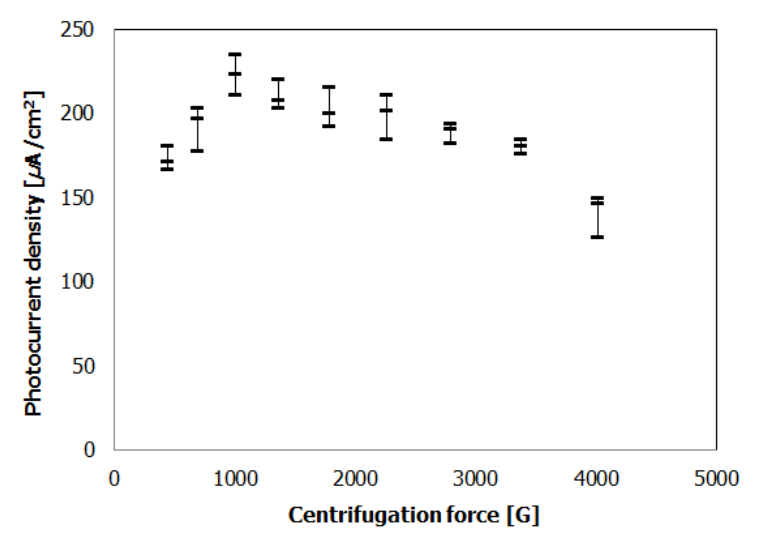

Fig. 8. Relationship between the photocurrent density and centrifugal force.

\section{CONCLUSIONS}

The results from this study demonstrated that it is possible to control phase separation by the use of the centrifugation method. In addition, this method can be effective for forming a continuous morphology in the direction of both electrodes. This centrifugation method was utilized with n- and p-type semiconducting materials with different specific gravities. We also obtained an ideal structure, which was suitable for charge transfer in the direction of both electrodes, by controlling the centrifugal force applied vertical to the electrode. A centrifugal force of approximately $1000 \mathrm{G}$ was suitable for obtaining the highest photocurrent density for the P3HT and Ti(IV) isopropoxide photoactive layer. By using the centrifugation method, a power conversion efficiency that was 2.2 times higher than that obtained using the conventional spin-coating method could be obtained.

\section{ACKNOWLEDGMENT}

The contribution of Mr. Iwao Kikuchi especially during the $\mathrm{J}-\mathrm{V}$ measurement work is highly commended. I really appreciate your efforts.

\section{REFERENCES}

[1] S. C. Price, A. C. Stuart, L. Yang, H. Zhou, and W. You, "Fluorine substituted conjugated polymer of medium band gap yields $7 \%$ efficiency in polymer-fullerene solar cells," J. Am. Chem. Soc., vol. 133, no. 12, pp. 4625-4631, March 2011.

[2] Y. Y. Liang, Z. Xu, J. B. Xia, S. T. Tsai, Y. Wu, G. Li, C. Ray, and L. P. Yu, "For the bright future - Bulk heterojunction polymer solar cells with power conversion efficiency of 7.4\%," Adv. Mater., vol. 22, no. 20, pp. E135-E138, May 2010.

[3] T.-Y. Chu, J. Lu, S. Beaupré, Y. Zhang, J.-R. M. Pouliot, S. Wakim, J. Zhou, M. Leclerc, Z. Li, J. Ding, and Y. Tao, "Bulk heterojunction solar cells using thieno[3,4-c]pyrrole-4,6-dione and dithieno[3,2-b:2',3'-d]silole copolymer with a power conversion efficiency of 7.3\%," J. Am. Chem. Soc., vol. 133, no. 12, pp. 4250-4253, Mar. 2011.

[4] H. Zhou, L. Yang, A. C. Stuart, S. C. Price, S. Liu, and W. You, "Development of fluorinated benzothiadiazole as a structural unit for a polymer solar cell of 7\% efficiency," Angew. Chem., Int. Ed., vol. 50 no. 13, pp. 2995-2998, March 2011.

[5] R. A. J. Janssen and J. Nelson, "Factors limiting device efficiency in organic photovoltaics," Adv. Mater., vol. 25, pp. 1847-1858, Dec. 2012.

[6] J. Nelson, "Polymer: Fullerene bulk heterojunction solar cells," Mater. Today, vol. 14, no. 10, pp. 462-470, October 2011.

[7] Z. He, C. Zhong, S. Su, M. Xu, H. Wu, and Y. Cao, "Enhanced power-conversion efficiency in polymer solar cells using an inverted device structure," Nat. Photonics, vol. 6, pp. 591-595, Aug. 2012.

[8] C. G. Shuttle, R. Hamilton, B. C. O’Regan, J. Nelson, and J. R. Durrant, "Charge-density-based analysis of the current-voltage response of polythiophene/fullerene photovoltaic devices," Proc. Natl. Acad. Sci. USA, pp. 16448-16452, vol. 107, 2010.

[9] G. F. A. Dibb, T. Kirchartz, D. Credgington, J. R. Durrant, and J. Nelson, "Analysis of the relationship between linearity of corrected photocurrent and the order of recombination in organic solar cells," $J$. Phys. Chem. Lett., vol. 2, no. 19, pp. 2407-2411, Sept. 2011.

[10] A. Maurano, C. G. Shuttle, R. Hamilton, A. M. Ballantyne, J. Nelson, W. Zhang, M. Heeney, and J. R. Durrant, "Transient optoelectronic analysis of charge carrier losses in a selenophene: Fullerene blend solar cell," J. Phys. Chem. C, vol. 115, no. 13, pp. 5947-5957, March 2011.

[11] M. Granström, K. Petritsch, A. C. Arias, A. Lux, M. R. Andersson, and R. H. Friend, "Laminated fabrication of polymeric photovoltaic diodes," Nature, vol. 395, pp. 257-260, Sept. 1998

[12] Y. Yuan, T. Michinobu, J. Oguma, T. Kato, and K. Miyake, "Attempted inversion of semiconducting features of platinum polyyne polymers: A new approach for all-polymer solar cells," Macromol. Chem. Phys., vol. 214, no. 13, pp. 1465-1472, July 2013.

[13] S. K. Das, K. Abe, K. Yoshino, Y. Ogomi, S. S. Pandey, and S. Hayase, "Controlling the processable $\mathrm{ZnO}$ and polythiophene interface for dye-sensitized thin film organic solar cells," Thin Solid Films, vol. 536, pp. 302-307, June 2013

[14] P. A. Hal, M. M. Wienk, J. M. Kroon, W. J. H. Verhees, L. H. Slooff, W. J. H. Gennip, P. Jonkheijm, and R. A. J. Janssen "Photoinduced electron transfer and photovoltaic response of a MDMO-PPV:TiO $\mathrm{Ti}_{2}$ bulk-heterojunction," Adv. Mater., vol. 15, no. 2, pp. 118-121, January 2003. 
[15] N. Hagiwara, K. Baiju, T. Kato, Y. Nasu, S. Izawa, K. Tanaka, and A. Kato, "Highly efficient organic-inorganic solar cell," in Proc. the $25^{\text {th }}$ International Symposium on Transport Phenomena, Nov. 2014, p. 10.

[16] M. Campoy-Quiles, T. Ferenczi, T. Agostinelli, P. G. Etchegoin, T. D. Anthopoulos, P. N. Stavrinou, D. D. C. Bradley, and J. Nelson, "Morphology evolution via self-organization and lateral and vertical diffusion in polymer: fullerene solar cell blends," Nature materials, vol. 7, no. 2, pp. 158-164, Jan. 2008.

[17] B. Schmidt-Hansberg, M. Sanyal, M. F. G. Klein, M. Pfaff, N. Schnabel, S. Jaiser, A. Vorobiev, E. Müller, A. Colsmann, P. Scharfer, D. Gerthsen, U. Lemmer, E. Barrena, and W. Schabel, "Moving through the phase diagram: Morphology formation in solution cast polymer-fullerene blend films for organic solar cells," ACS Nano, vol. 5, no. 11, pp. 8579-8590, October 2011.

[18] M. A. Hammamiab, M. Krifaa, and O. Harzallahb, "Centrifugal force spinning of PA6 nanofibers - Processability and morphology of solution-spun fibers," The Journal of The Textile Institute, vol. 105, no. 6, pp. 637-647, Jan. 2014.

[19] S. E. Hadad, H. Sato, E. Miura, and Y. Watanabe, "Fabrication of $\mathrm{Al}-\mathrm{Al}_{3} \mathrm{Ti} / \mathrm{Ti}_{3} \mathrm{Al}$ functionally graded materials under a centrifugal force," Materials, vol. 3, no. 9, pp. 4639-4656, Sept. 2010.

[20] S. R. Cowan, A. Roy, A. J. Heeger, "Recombination in polymer-fullerene bulk heterojunction solar cells," Phys. Rev. B, vol. 82, p. 245207, Dec. 2010.

[21] R. A. Street, S. Cowan, and A. J. Heeger, "Experimental test for geminate recombination applied to organic solar cells," Phys. Rev. B, vol. 82, p. 121301, September 2010.

[22] C. G. Shuttle, A. Maurano, R. Hamilton, B. O'Regan, J. C. de Mello, and J. R. Durrant, "Charge extraction analysis of charge carrier densities in a polythiophene/fullerene solar cell: Analysis of the origin of the device dark current," Appl. Phys. Lett., vol. 93, no. 18, 183501, November 2008.

[23] C. G. Shuttle, B. O’Regan, A. M. Ballantyne, J. Nelson, D. D. C. Bradley, J. de Mello, and J. R. Durrant, "Experimental determination of the rate law for charge carrier decay in a polythiophene: Fullerene solar cell," Appl. Phys. Lett., vol. 92, p. 093311, March 2008.

[24] M. Reyes-Reyesa, R. López-Sandovalb, J. Arenas-Alatorrec, R. Garibay-Alonsob, D. L. Carrolld, and A. Lastras-Martinez, "Methanofullerene elongated nanostructure formation for enhanced organicsolar-cells," Thin Solid Films, vol. 516, no. 1, pp. 52-57, November 2007.

[25] C. G. Shuttle, B. O’Regan, A. M. Ballantyne, J. Nelson, D. D. C. Bradley, and J. R. Durrant, "Bimolecular recombination losses in polythiophene: Fullerene solar cells," Phys. Rev. B, vol. 78, p. 113201, September 2008

[26] D. Mori, H. Benten, H. Ohkita, S. Ito, and K. Miyake, "Polymer/polymer blend solar cells improved by using high-molecular-weight fluorene-based copolymer as electron acceptor," ACS Appl. Mater. Interfaces, vol. 4, no. 7, pp. 3325-3329, June 2012

[27] J. You, L. Dou, K. Yoshimura, T. Kato, K. Ohya, T. Moriarty, K. Emery, C.-C. Chen, J. Gao, G. Li, and Y. Yang, "A polymer tandem solar cell with $10.6 \%$ power conversion efficiency," Nature Commun., vol. 4, p. 1446, February 2013.

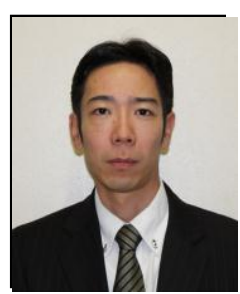

Takehito Kato is a lecturer of mechanical engineering, Oyama College of National Institute of Technology, Japan. He received his PhD in 2007 at Kyushu Institute of Technology, Japan. Then, he had been a researcher in Sumitomo Chemical Co., Ltd. during 2007-2012. He is a member of the Japan Society of Mechanical Engineers, the Electrochemical Society of Japan and the Japan Society of Applied Physics. Since 2012, He has been a senator of Kanto branch in the Japan Society of Mechanical Engineers. And he has been an editor of International Journal of Sensing, Computing \& Control. His current research interests focus on organic-inorganic hybrid materials and devices. 